\title{
Characterization of cytarabine-resistant leukemic cell lines established from five different blood cell lineages using gene expression and proteomic analyses
}

\author{
EIJU NEGORO ${ }^{1}$, TAKAHIRO YAMAUCHI ${ }^{1}$, YOSHIMASA URASAKI ${ }^{1}$, \\ RIE NISHI $^{1}$, HIROKI HORI ${ }^{2}$ and TAKANORI UEDA ${ }^{1}$ \\ ${ }^{1}$ Department of Hematology and Oncology, Faculty of Medical Sciences, University of Fukui, \\ 23-3 Shimoaizuki, Matsuoka, Eiheiji, Fukui 910-1193; ${ }^{2}$ Department of Pediatrics, \\ Mie University School of Medicine, 1577 Kurimamachiya, Tsu, Mie 514-8507, Japan
}

Received November 2, 2010; Accepted December 21, 2010

DOI: 10.3892/ijo.2011.933

\begin{abstract}
Cytarabine (ara-C) is the key drug for treatment of acute myeloid leukemia. Since intracellular cytarabine triphosphate (ara-CTP) is an active metabolite of ara-C, factors that reduce the amount of ara-CTP are known to induce drug resistance. However, these factors do not fully explain the development of resistance to ara-C. The present study was conducted to search for new candidate ara- $\mathrm{C}$ resistance factors, including those that are unrelated to ara-CTP production. For this purpose, we newly established five ara-C-resistant leukemic clones from different blood cell lineage leukemic cell lines (HL-60, K562, CEM, THP1 and U937). The resistant subclones were 5-58-fold more ara-C-resistant than their parental counterparts. All of the ara-C-resistant subclones, except for ara-C-resistant CEM cells, displayed alteration of ara-CTPrelated factors such as ara-C membrane transport capacity, deoxycytidine kinase activity or cytosolic nucleotidase II activity. To identify new candidate factors, we used two comprehensive approaches: DNA microarray and proteome analyses. The DNA microarray analysis revealed eight genes (C19orf2, HSPA8, LGALS1, POU4F3, PSAP, AKT1, MBC2 and CACNA2D3) that were altered in all five ara-C-resistant lines compared to parental cells. Both proteome and DNA microarray analyses further detected a reduced protein level of stathmin 1 in the ara-C-resistant CEM subclone compared to its parental line. Thus, the present findings suggested the involvement of novel multiple mechanisms in mediating the ara-C resistance of leukemic cells. The role of some of these molecules in resistance is still unclear.
\end{abstract}

Correspondence to: Dr Takahiro Yamauchi, Department of Hematology and Oncology, Faculty of Medical Sciences, University of Fukui, 23-3 Shimoaizuki, Matsuoka, Eiheiji, Fukui 910-1193, Japan

E-mail: tyamauch@u-fukui.ac.jp

Key words: ara-C, resistance, DNA microarray analysis, proteome analysis, leukemia

\section{Introduction}

Nucleoside analogues constitute an important family of anti-neoplastic agents that are used in the treatment of hematological malignancies $(1,2)$. One of these analogues, the sugar-modified pyrimidine analog cytarabine (1- $\beta$-D-ara binofuranosylcytosine; ara-C) is the key agent for the treatment of acute myeloid leukemia (AML) (2). The standard induction therapy, consisting of conventional doses of ara- $\mathrm{C}$ for 7 days plus anthracycline for 3 days, provides over $70 \%$ remission rates for AML (3-5). Nevertheless, only $40 \%$ of the patients are long-term survivors; most relapse with the development of drug resistance. Thus, overcoming ara- $\mathrm{C}$ resistance is essential for the improvement of clinical outcomes.

For ara-C to function as an anti-tumor agent, it must first be transported into leukemic cells by membrane transporters such as the human Equilibrative Nucleoside Transporter 1 (hENT1) (6). Inside the cell, ara-C is phosphorylated by the rate-limiting enzyme deoxycytidine kinase $(\mathrm{dCK})$ to ara- $\mathrm{C}$ monophosphate, then to ara- $\mathrm{C}$ diphosphate by deoxycytidine monophosphate kinase, and eventually to ara-C triphosphate (ara-CTP), an active metabolite of ara-C, by nucleoside diphosphate kinase (2). Ara-CTP is then incorporated into DNA strands during the $S$ phase of the cell cycle, thereby inhibiting DNA synthesis (7-10). Since ara-C cytotoxicity depends on ara-CTP incorporation into DNA and therefore depends on ara-CTP concentration, ara-CTP levels thus represent an index of ara-C cytotoxicity. Intracellular ara-CTP levels also have clinical value since a correlation between the intracellular pharmacokinetics of ara-CTP and response to ara-C therapy has been established (11-14).

Acquired resistance to anticancer agents is a major obstacle to successful cancer treatment, and overcoming resistance to ara-C would offer new strategies for the treatment of AML. Because the production of ara-CTP depends on the cellular activation of ara- $\mathrm{C}$, cellular factors that can regulate ara- $\mathrm{C}$ activation play a role in ara- $\mathrm{C}$ resistance (11-16). Ara- $\mathrm{C}$ catabolism results from rapid deamination by cytidine deaminase to the non-toxic metabolite ara-U (2), while cytosolic 5'-nucleotidase II (cN-II) dephosphorylates ara-CMP (7), 
thereby preventing the production of the active form. Reduced hENT1 activity, decreased dCK activity, and increased cN-II activity are so far the only markers that have been shown to be clinically significant markers of the therapeutic efficacy of ara-C-based chemotherapy (17-20). However, not all the mechanisms of resistance to ara- $\mathrm{C}$ can be explained by these factors. Therefore, a more comprehensive technique is necessary to further clarify the biochemical and molecular mechanisms underlying ara-C resistance.

In the present study, we established five ara-C-resistant subclones from five different blood lineage leukemic cell lines. These ara-C-resistant subclones were then extensively evaluated regarding their mechanisms of ara-C resistance. First, the level and activity of known ara-C-related factors such as hENT1, dCK, and cN-II were examined using conventional methods. Next, DNA microarray analysis was performed to identify novel resistance-related genes (21-23). For this analysis, the gene expression profiles of all five ara-C-resistant subclones were compared with their parental counterparts. Finally, for further comprehensive investigation, proteome analysis of one pair of ara-C-sensitive and ara-C-resistant cells was carried out.

\section{Materials and methods}

Chemicals and reagents. Ara-C was a generous gift from Nippon Shinyaku Co. (Kyoto, Japan). Mitoxantrone hydrochloride (MIT) was from Takeda Co. (Osaka, Japan). Vincristine (VCR) and gemcitabine ( $\mathrm{dFdC})$ were kindly provided by Nippon Kayaku Co. (Tokyo, Japan), and Eli Lilly Co. (Indianapolis, IN), respectively. All other materials were of the highest grade commercially available. $\left[5-{ }^{3} \mathrm{H}\right]$-ara-C $(30$ $\mathrm{Ci} / \mathrm{mmol}$ ) was purchased from Daiichi Pure Chemicals (Tokyo, Japan). [8- $\left.{ }^{14} \mathrm{C}\right]-\mathrm{IMP}(45-60 \mathrm{mCi} / \mathrm{mmol})$ was purchased from Moravek Biochemicals (Brea, CA, USA).

Cell culture. Human monocytic leukemia THP-1 cells, human T-lymphoblastic leukemia CCRF-CEM cells, human monocytic leukemia U937 cells, human promyelocytic leukemia HL60 cells, and human erythroleukemia K562 cells were purchased from the Health Science Research Resource Bank (Japan Health Science Foundation, Tokyo, Japan). The cells were cultured in RPMI-1640 media supplemented with $10 \%$ heat-inactivated fetal calf serum, and were maintained in a $5 \% \mathrm{CO}_{2}$-humidified atmosphere at $37^{\circ} \mathrm{C}$.

Development of ara-C-resistant variants. To develop ara-C resistant variants, cells of the five different cell lines above were treated with increasing concentrations of ara-C (1 nM-1 $\mu \mathrm{M})$. The cultures were observed daily and were passaged using gradually increasing concentrations of ara-C. When their doubling time in the presence of $1 \mu \mathrm{M}$ ara-C was almost the same as that of the parent cell lines in the absence of ara-C, the cells were cloned using the limiting dilution method. The ara-C-resistant subclones HL-60/ara-C, K562/ara-C, CEM/ ara-C, U937/ara-C, and THP/ara-C were developed from parental HL-60, K562, CEM, U937, and THP clones, respectively.

Proliferation assay. To evaluate the proliferative activity of each cell line, the sodium 3'-[1-(phenylaminocarbonyl)-3,4- tetrazolium]-bis (4-methoxy-6-nitro) benzene sulfonic acid hydrate (XTT) assay was performed according to the manufacturer's instructions (Roche, Indianapolis, IN, USA) with slight modifications (24).

Western blotting. Western blot analysis was performed to determine the protein levels of dCK. Cell lysates were prepared from $1 \times 10^{7}$ cells using the lysis buffer. Lysates were resuspended in loading buffer, subjected to sodium dodecyl sulfate-polyacrylamide gel electrophoresis on a $10 \%$ acrylamide gel, and electrophoretically transferred onto Immobilon-P membranes (Millipore, Billerica, MA, USA). The membranes were probed with primary and subsequently secondary antibodies using standard techniques. The enhanced chemiluminescence (ECL) detection kit (Cell Signaling Technology, Beverly, MA, USA) and Hyperfilm ECL (AmershamBiosciences UK,LittleChalfont,Buckinghamshire, UK) were used to visualize antibody-protein binding. The antibody against dCK, which was developed in the Department of Pediatrics, Mie University School of Medicine (25), and an anti-actin antibody (Sigma, St Louis, MO, USA), were used as the primary antibodies.

Nucleoside transport capacity. To evaluate the capacity of membrane nucleoside transporters, nucleoside analog uptake was quantified in all cell lines using the method of Wiley et al with slight modifications $(6,24)$. Non-facilitated drug uptake was determined in the presence of $3 \mu \mathrm{M}$ nitrobenzylthioinosine (Sigma), which interferes with the function of membrane nucleoside transporters. The capacity of the transporter was determined as the difference between drug uptake in the absence and presence of nitrobenzylthioinosine.

Measurement of $d C K$ and $c N-I I$ activity. The activity of $\mathrm{dCK}$ was assayed as previously described with slight modifications (24). Briefly, crude enzyme was obtained by sonication of cells suspended in $100 \mu \mathrm{l}$ of $50 \mathrm{mM}$ Tris- $\mathrm{HCl}$ (pH 8.0) containing $50 \%$ glycerol, and the solution was then clarified by centrifugation $\left(100,000 \mathrm{x} \mathrm{g}\right.$ for $60 \mathrm{~min}$ at $\left.4^{\circ} \mathrm{C}\right)$. The enzyme $(20 \mu \mathrm{l})$ was incubated for $30 \mathrm{~min}$ at $37^{\circ} \mathrm{C}$ in a reaction buffer (total volume, $60 \mu \mathrm{l}$ ) containing $20 \mu \mathrm{M}\left[5-{ }^{3} \mathrm{H}\right]$ ara-C, $40 \mathrm{mM}$ Tris- $\mathrm{HCl}$ (pH 8.0), $10 \mathrm{mM} \mathrm{MgCl}$, $10 \mathrm{mM}$ ATP, $12.5 \mathrm{mM}$ dithiothreitol, and $1 \mathrm{mM}$ tetrahydrouridine. The reaction was terminated by placing the sample in an ice bath. A $10-\mu 1$ aliquot of the sample was spotted on a cellulose thin-layer chromatography sheet (Polygram CEL 300 UV254, $20 \mathrm{~cm}$ x $20 \mathrm{~cm}$ x $0.1 \mathrm{~mm}$, Macherey-Nagel, Postfach, Düren, Germany), and developed for $3 \mathrm{~h}$ in a solvent (water:2-propanol:acetic acid = 1:2:2, vol/ vol). The plate was cut into $1-\mathrm{cm}$ strips, which were then placed in a scintillation vial filled with $10 \mathrm{ml}$ of Clear-sol 1 (Nacalai Tesque, Kyoto, Japan). Radioactivity was counted on the following day. Enzyme activity was expressed in picomoles per hour per mg protein.

The activity of $\mathrm{cN}-\mathrm{II}$ was measured as previously described with slight modifications (24). The crude enzyme (20 $\mu \mathrm{l})$, obtained as described for $\mathrm{dCK}$, was mixed with reaction buffer (total volume, $60 \mu \mathrm{l}$ ) that contained $50 \mathrm{mM}$ imidazole (pH 7.5), $50 \mathrm{mM} \mathrm{NaCl}, 10 \mathrm{mM} \mathrm{MgCl}_{2}, 0.5 \mathrm{~g} / 1$ bovine serum albumin, $0.2 \mathrm{mM} \alpha, \beta$-methylene adenosine diphosphate (an inhibitor of the activity of membrane-bound 5'-ecto-nucleotidase), 
Table I. Sensitivity of 5 leukemic cell lines and their ara-C-resistant counterparts to anticancer agents.

\begin{tabular}{|c|c|c|c|c|c|}
\hline & Ara-C (RR) & $\mathrm{dFdC}(\mathrm{RR})$ & MIT (RR) & VCR (RR) & CPT-11 (RR) \\
\hline HL-60 & 0.2 & 0.01 & 0.01 & 0.02 & 0.02 \\
\hline HL-60/ara-C & $1.1(6.0)$ & $0.07(7.0)$ & $0.02(1.9)$ & $0.02(1.0)$ & $0.02(1.0)$ \\
\hline K562 & 0.1 & 0.02 & 0.01 & 0.02 & 0.01 \\
\hline K562/ara-C & $1.5(15)$ & $0.03(1.5)$ & $0.02(2.0)$ & $0.02(1.0)$ & $0.01(1.0)$ \\
\hline CEM & 0.1 & 0.1 & 0.02 & 0.01 & 0.01 \\
\hline CEM/ara-C & $0.5(5.0)$ & $0.4(4.0)$ & $0.03(1.5)$ & $0.01(1.0)$ & $0.01(1.0)$ \\
\hline THP-1 & 3 & 0.3 & 0.4 & 0.05 & 0.2 \\
\hline THP-1/ara-C & $174(58)$ & $58(190)$ & $0.6(1.5)$ & $0.05(1.0)$ & $0.2(1.0)$ \\
\hline U937 & 0.5 & 0.2 & 0.02 & 0.01 & 0.02 \\
\hline U937/ara-C & $2.6(5.0)$ & $0.4(2.0)$ & $0.02(1.0)$ & $0.01(1.0)$ & $0.02(1.0)$ \\
\hline
\end{tabular}

$\mathrm{RR}$, relative resistance calculated as the ratio of the $\mathrm{IC}_{50}$ of ara-C resistant cells relative to that of the parental cells. Ara-C, cytarabine, $\mathrm{dFdC}$; gemcitabine; MIT, mitoxantrone hydrochloride; VCR, vincristine; CPT-11, irinotecan. P $\leq 0.05$.

$5 \mathrm{mM} \beta$-glycerophosphate, $100 \mu \mathrm{M}$ EGTA, and $200 \mu \mathrm{M}$ IMP and $\left[8-{ }^{14} \mathrm{C}\right]$-IMP. Three millimolar ATP was added to activate $\mathrm{cN}$-II. The reaction was allowed to continue for $30 \mathrm{~min}$ at $37^{\circ} \mathrm{C}$ and was terminated by placing the sample in an ice bath. A $10-\mu 1$ aliquot of the sample was applied to a thin-layer chromatographic sheet as described above. Enzyme activity was expressed as picomoles per hour per mg protein.

Gene-expression profiling using DNA microarray analysis. Total RNA was isolated from $3 \times 10^{6}$ cells per sample and was assessed using gel electrophoresis. Cy-3 labeled total RNA from parental cells (THP-1, K562, HL60, CEM, U937) and Cy-5 labeled total RNA from the corresponding ara-C-resistant cells (THP-1/ara-C, K562/ara-C, HL60/ara-C, CCRF-CEM/ ara-C, U937/ara-C) were hybridized on microarrays of complementary DNA that contained 20,173 elements (22K), (Human1A ver2. Oligo, Agilent Technologies, Palo Alto, CA, USA) that were prepared by Hokkaido System Science Co., Ltd. (Sapporo, Japan). Hybridized images were scanned using a fluorescence laser scanning device (DNA Microarray Scanner; Agilent). Data were retrieved as $\log 10$ (Cy5/Cy3). The final data were expressed as the fold-change in the gene expression value between the ara-C-resistant subclone and its corresponding parental cell line.

Proteome analysis [two-dimensional difference gel electrophoresis (DIGE) and mass spectrometry]. For DIGE minimal labeling, $10 \mu \mathrm{g}$ of each of protein sample was mixed with $80 \mathrm{pmol}$ CyDye (GE Healthcare Bio-Sciences, Piscataway, NJ, USA) and incubated on ice in the dark for $30 \mathrm{~min}$. Proteins extracted from parental and ara-C-resistant cells of each cell line were labeled with $\mathrm{Cy} 3$ and $\mathrm{Cy} 5$, respectively, and were then mixed with a Cy2-labeled internal standard and run on the same gel. The total proteins $(30 \mu \mathrm{g})$ were mixed and denatured in sample buffer (8 M urea, 2\% CHAPS, 2-4\% IPG buffer, 1.2-2.4\% Destreak Solution, 2.8-5.6 mg/ml DTT, Bromophenol blue), and were then rehydrated with Immobiline DryStrip ( $\mathrm{pH} 3.0$ 10.0, $7 \mathrm{~cm}$; GE Healthcare Bio-Sciences) for the first-dimension isoelectric focusing. Thereafter, the strips were applied to the second dimension 5-20\% sodium dodecyl sulfate-polyacrylamide gel electrophoresis (SDS-PAGE) (SuperSep, Wako, Osaka, Japan) using an electrophoresis system. The Cye-dye labeled gels were visualized using a Typhoon Trio bioimaging analyzer (GE Healthcare Bio-Sciences). Inter-gel matching and statistical analysis were performed using Progenesis PG240 (PerkinElmer, Wellesley, MA, USA). The proteins separated by 2-DE were stained with Coomassie blue (SeePico CBB Stain kit, Benebiosis, Seoul, Korea). The protein spots of interest were manually excised from the gel, followed by destaining, reduction, alkylation, and digestion with trypsin (Roche, Basel, Switzerland), and were then subjected to MALDI-TOF/MS (Autoflex, Bruker Daltonics, Bremen, Germany) for peptide mass fingerprinting. Protein identification was carried out using a Mascot search (Matrix Science, Boston, MA, USA) by sending the data of peptide mass fingerprinting as a query.

Statistical analyses. All statistical analyses were performed using Microsoft Excel 2007 software (Microsoft, Redmond, WA, USA). All graphs, linear regression lines, and curves were generated using GraphPad Prism software (version 5.0) (GraphPad Software, Inc., San Diego, CA, USA). Values of $\mathrm{P} \leq 0.05$ were considered statistically significant.

\section{Results}

Establishment of ara-C-resistant leukemic cells. An ara-Cresistant subclone was established from each of five different leukemic cell lines. The subclones were HL-60/ara-C, K562/ ara-C, CEM/ara-C, U937/ara-C, and THP1/ara-C, which were derived from the corresponding parental clones of HL60, K562, CEM, U937, and THP1, respectively. The XTT proliferation assay demonstrated that these variants were 5- to 58-fold more resistant to ara- $\mathrm{C}$ than their ara-C-sensitive parents (Table I). To determine if ara-C resistance was associated with cross-resistance to other agents, the cells were treated with various anticancer agents, and their proliferation was then assayed (Table I). Ara-C-resistant variants exhibited cross- 
(A)

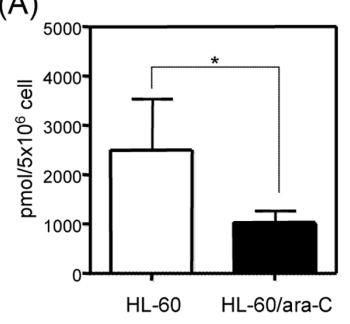

(D)

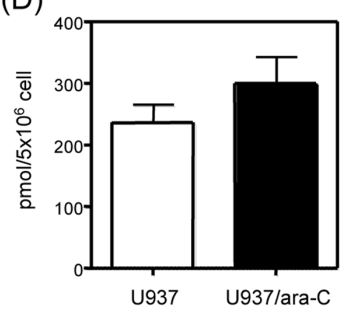

(B)

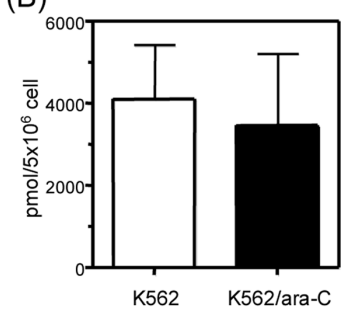

(E)

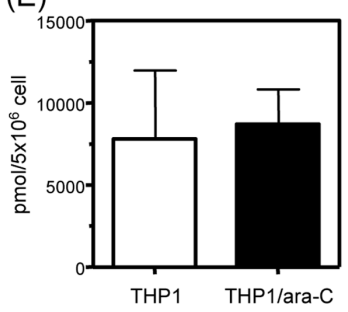

(C)

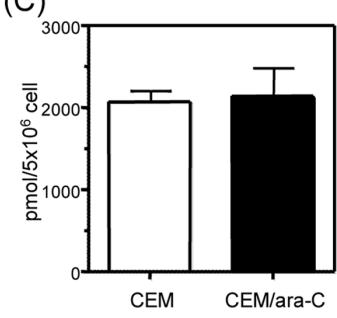

Figure 1. Transport of ara- $\mathrm{C}$ into parental and ara- $\mathrm{C}$ resistant cells. Membrane nucleoside transport of ara- $\mathrm{C}$ by the indicated parental and ara- $\mathrm{C}$ resistant (ara-C) cells was assessed by pulsing the cells with $0.32 \mu \mathrm{M}$ tritiated ara-C for $60 \mathrm{sec}$, followed by quantification of cellular drug uptake by scintillation counting. Each value represents the mean $\pm \mathrm{SD}$ of at least three independent experiments. ${ }^{*} \mathrm{p} \leq 0.05$.

(A)

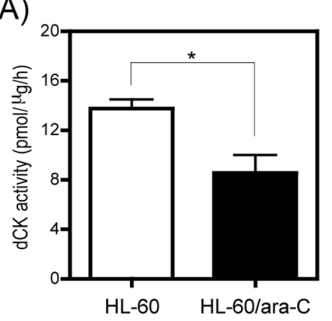

(D)

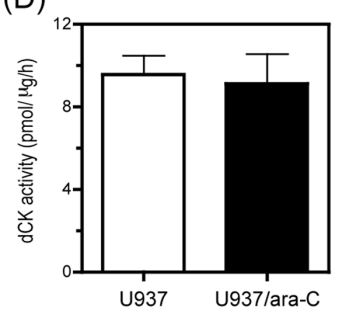

(B)

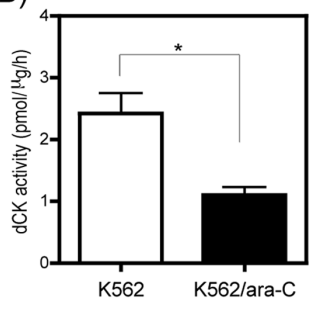

(E)

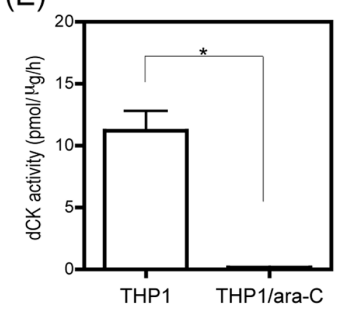

(C)

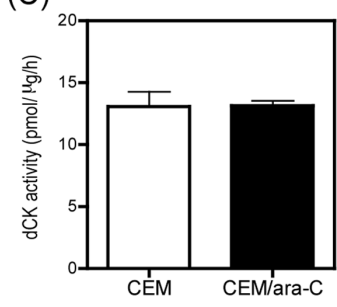

Figure 2. dCK activity of pairs of parental and ara-C resistant cell lines (A-E). The activity of dCK was determined in cell extracts of each subclone and its parental cell line using ara- $\mathrm{C}$ as a substrate. Each value represents the mean $\pm \mathrm{SD}$ of at least three independent experiments. * $\mathrm{p} \leq 0.05$.

resistance to a nucleoside analog, $\mathrm{dFdC}$, which is similar to ara-C, but did not show resistance against MIT, VCR or CPT-11 (Table I). Thus, the results suggested that the resistant nature of the subclones established here was specific to ara-C and similar nucleoside analogs.

Membrane nucleoside transport capacity. For ara-C to exert anti-tumor activity, it must first be transported into cells. We therefore determined whether the ara-C resistance of the established clones was related to an inability to transport ara-C into cells. When the cells were pulsed with $1 \mu \mathrm{M}$ tritiated ara-C, the drug was rapidly incorporated intracellularly by all five cell lines (Fig. 1). However, ara-C influx was significantly reduced in the HL-60/ara-C cells compared with the parental
HL-60 cells (Fig. 1A). Tritiated ara-C uptake was similar between the other ara-C-resistant subclones and their respective ara-C-sensitive parents (Fig. 1B-D). These results suggested that decreased membrane nucleoside transport capacity was associated with the development of ara- $\mathrm{C}$ resistance only in HL-60/ara-C cells.

Enzymatic activities of $d C K$ and $c N-I I$. To further elucidate the mechanisms of ara- $\mathrm{C}$ resistance in the established clones, we evaluated the enzymatic activity of $\mathrm{dCK}$ and $\mathrm{cN}-\mathrm{II}$, which are involved in the metabolism of ara- $\mathrm{C}$, leading to enhancement and inhibition respectively of ara- $\mathrm{C}$ antitumor effects. These enzymes are also one of the few clinically significant markers of the therapeutic efficacy of ara-C-based chemotherapy 


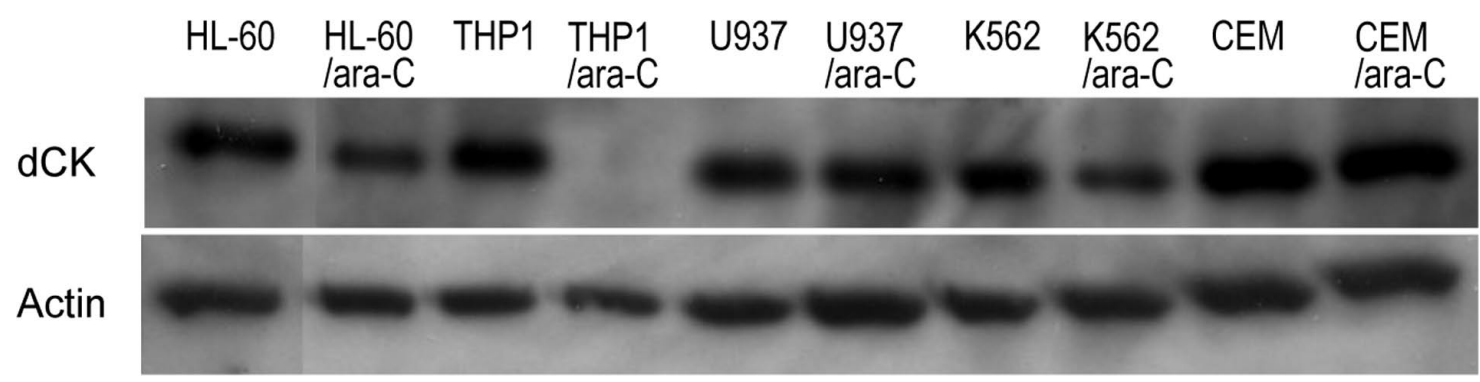

Figure 3. dCK protein expression in parental and ara-C resistant cell lines. Whole-cell lysates of the indicated cell lines were analyzed by Western blotting using an anti-dCK antibody. Actin was used as loading control.

(A)

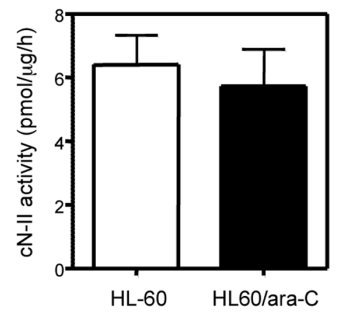

(D)

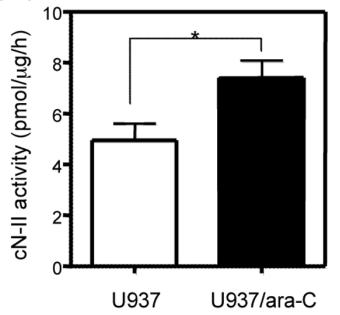

(B)

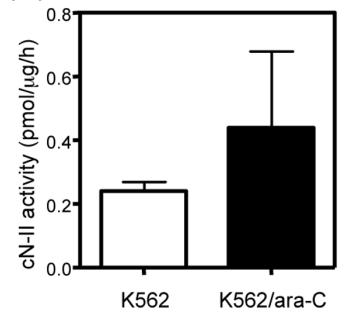

(E)

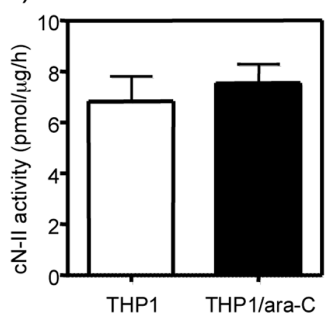

(C)

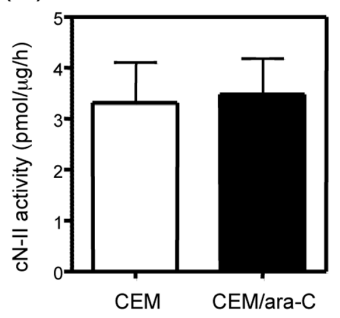

Figure 4. cN-II activity of paired parental and ara-C resistant cells (A-E). The activity of cN-II was determined in cell extracts of each subclone and its parental cell line using IMP as a substrate in the presence of ATP. Each value represents the mean \pm SD of at least three independent experiments. * $\leq 0.05$.

(15-18). The enzymatic activity of dCK was reduced in HL60/ ara-C cells, K562/ara-C cells, and THP1/ara-C cells compared to the respective parental cell lines (Fig. 2). A decrease in $\mathrm{dCK}$ activity would reduce phosphorylation of ara- $\mathrm{C}$ to ara- $\mathrm{C}$ monophosphate and consequently decrease ara-CTP production, thereby conferring ara-C resistance on the cells. The decreased $\mathrm{dCK}$ activity correlated with a decrease in the protein level of dCK (Fig. 3) in these cell lines as assessed by Western blotting. The enzymatic activity of cN-II was augmented in U937/ara-C cells (Fig. 4), suggesting enhanced dephosphorylation of ara-C monophosphate, which prevents the production of ara-CTP. The combined results therefore suggested that ara-C-related factors, including $\mathrm{dCK}$ and $\mathrm{cN}-\mathrm{II}$, were responsible for the development of ara-C resistance in the HL60/ara-C, K562/ ara-C, THP1/ara-C, and U937/ara-C cell lines, but not in the $\mathrm{CEM} / \mathrm{ara}-\mathrm{C}$ cell line.

Gene-expression profiles evaluated using DNA microarray analysis. For a more comprehensive analysis of genes involved in ara-C resistance, the gene-expression profiles of ara-Cresistant and ara-C-sensitive parental cell lines were compared for each of the five cell lines using DNA microarray analysis. Overall, hundreds of up-regulated or down-regulated genes were detected in each ara-C-resistant subclone. However, a significant alteration of two up-regulated genes and six downregulated genes in ara-C-resistant cells compared to parental cells was commonly detected in all of these cell lines (Table II), although the degree of the fold-change was relatively small in some of the clones. The impact of these genes on the development of ara-C resistance has not been evaluated in this study. However, these genes are known to be involved in apoptosis or survival pathways, or to function as a molecular chaperone (26-33). Such functions could be closely associated with cellular survival after drug treatment. The levels of the genes encoding hENT1, dCK and cN-II were unchanged, suggesting that these factors must be altered by post-transcriptional regulation in the ara-C-resistant clones. In summary, microarray analysis identified eight genes that play unknown roles in the development of resistance to ara-C.

Proteome analysis of CEM/ara-C cells. The clinically relevant markers of ara-C-based therapy, hENT1, dCK, and cN-II, are the factors most likely to be associated with the mechanisms of resistance of a given ara-C-resistant leukemic cell. Nevertheless, the activities of these factors were not altered in CEM/ ara-C cells, suggesting that CEM cells may have a different 


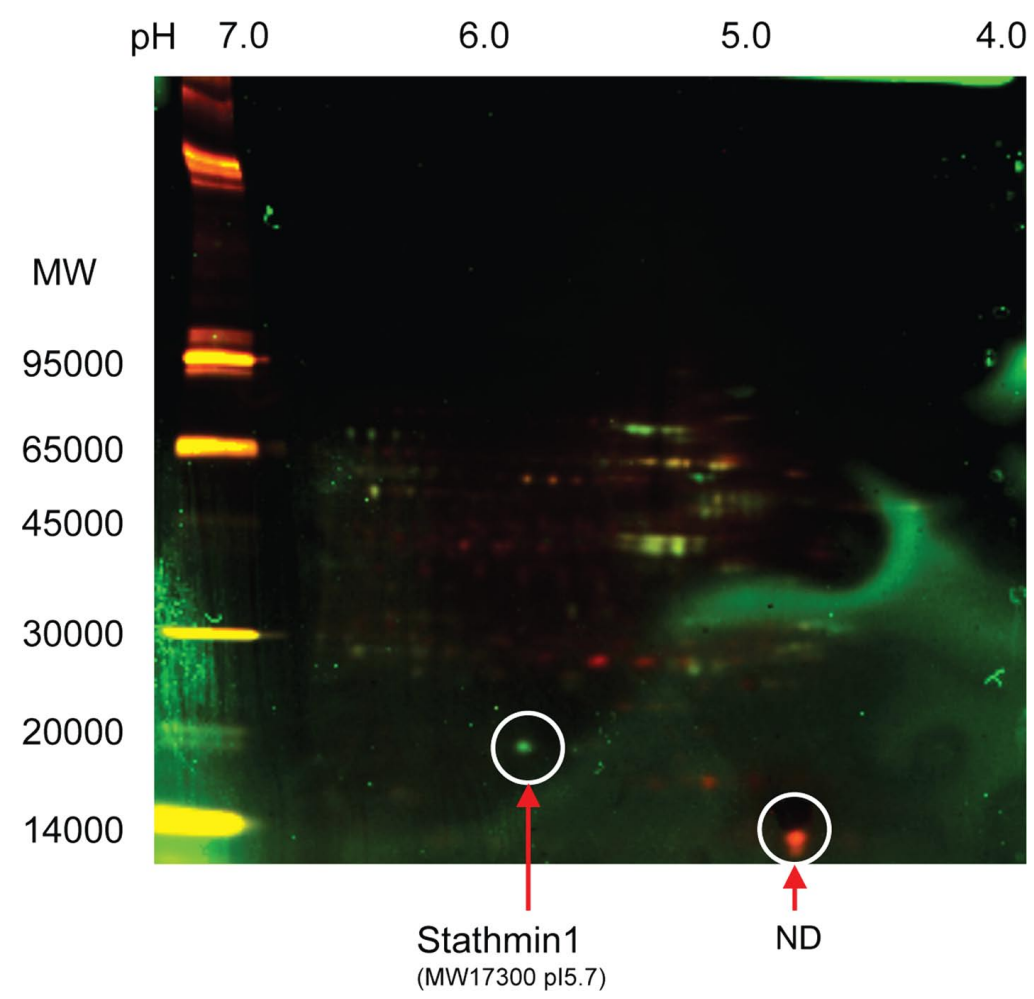

Figure 5. Proteome analysis of parental and ara-C-resistant CEM cells. Proteins extracted from CEM and CEM/ara-C cells were labeled with Cy3 and Cy5, respectively, and were analyzed by two-dimensional gel electrophoresis. Protein spots of interest, whose expression was up- (red) or down- (green) regulated in CEM/ara-C cells as compared to parental cells, were further evaluated by peptide mass fingerprinting. ND, not determined.

Table II. The common gene expression changes among 5 different ara-C-resistant cell lines.

\begin{tabular}{|c|c|c|c|c|c|c|}
\hline & & HL-60 & K562 & $\begin{array}{c}\text { CEM } \\
\text { Fold changes }\end{array}$ & U937 & THP-1 \\
\hline \multicolumn{7}{|c|}{ Up-regulated genes } \\
\hline C19orf2 & NM_003796 & 2.19 & 1.69 & 1.40 & 1.81 & 1.42 \\
\hline HSPA8 & NM_006597 & 1.38 & 3.49 & 1.46 & 1.30 & 2.04 \\
\hline \multicolumn{7}{|c|}{ Down-regulated genes } \\
\hline LGALS1 & NM_002305 & 0.71 & 0.40 & 0.56 & 0.43 & 0.49 \\
\hline POU4F3 & NM_002700 & 0.42 & 0.57 & 0.57 & 0.63 & 0.61 \\
\hline PSAP & NM_002778 & 0.49 & 0.37 & 0.60 & 0.69 & 0.79 \\
\hline AKT1 & NM_005163 & 0.72 & 0.75 & 0.64 & 0.71 & 0.74 \\
\hline MBC2 & NM_015292 & 0.69 & 0.79 & 0.67 & 0.66 & 0.73 \\
\hline CACNA2D3 & NM_018398 & 0.33 & 0.34 & 0.40 & 0.54 & 0.39 \\
\hline
\end{tabular}

Fold-change values represent mRNA levels for ara-C-resistant cells relative to those of their parent counterparts.

mechanism for mediating ara- $\mathrm{C}$ resistance. Although the genes identified by microarray analysis in all of the cell lines may also contribute to the resistance of CEM cells, it is possible that CEM cells also have specific mechanisms of ara-C resistance. Therefore, to further comprehensively analyze factors involved in ara-C resistance in CEM cells, we performed proteome analysis of CEM and CEM/ara-C cells. The image obtained from this analysis showed one green spot and one red spot in CEM/ara-C cells, indicating a protein with a reduced and an enhanced expression level, respectively, compared to the parental cells (Fig. 5). The green spot was identified as stathmin1, whereas the identity of the red one has not yet been determined. The gene expression level of stathmin1 was also reduced in the CEM/ara-C cells as compared to the parental cells when assayed by DNA microarray analysis (data not shown). Because stathmin1 is known to be a proliferation- 
associated or cell cycle-regulating protein $(34,35)$, it could be involved in mechanisms of resistance to the cell cycle-specific agent ara-C.

\section{Discussion}

The present study was conducted to search for new candidate molecules that might be associated with the development of resistance to ara-C. Our search involved DNA microarray analysis of five pairs of ara-C resistant and parental clones as well as proteome analysis of one pair that appeared to have a novel ara-C-resistant mechanism. Our findings suggested the involvement of multiple mechanisms in the acquired resistance to ara-C.

For our study, we isolated five ara-C-resistant leukemia cell lines, HL-60/ara-C, K562/ara-C, /CEM/ara-C, U937/ara-C, and THP-1/ara-C (Table I). Only one of the five ara-C-resistant subclones (CEM/ara-C) did not show a change in known ara-CTP-related factors (hENT1, dCK, cN-II) compared to parental cell lines (Figs. 1-4). Thus, HL-60/ara-C cells showed a decrease in ara-C membrane transport capacity and $\mathrm{dCK}$ activity (Figs. 1 and 2), K562/ara-C cells and THP-1/ara-C cells exhibited decreased dCK activity and U937/ara-C cells displayed enhanced cN-II activity (Figs. 2-4). The fact that four of the five cell lines had alterations in ara-CTP-related factors is in agreement with other studies that intracellular activation of ara-C to ara-CTP is the most crucial factor for determination of cellular sensitivity to ara-C (11-14,18). Our study did not detect an increase in total cellular glutathione, which we previously identified in leukemic cells that were resistant to both ara-C and daunorubicin (36), suggesting that the mechanism of resistance to ara- $\mathrm{C}$ of the clones established here might be ara-C-specific. Our results further suggested that factors that have not ever been determined can also induce ara-C resistance because the CEM/ara-C cells were ara-C-resistant but showed no apparent changes in the membrane transport capacity, dCK, and $\mathrm{cN}-\mathrm{II}$. Thus, these data suggested multiple mechanisms of ara-C resistance, including new candidate factors whose roles are not fully understood.

The DNA microarray technique has recently been applied to cancer cells, including leukemia cells, to generate a list of genes that might predict response to chemotherapy and/or the prognosis of patients $(21-23,37,38)$. Microarray profiling is a powerful tool that has the advantage of high throughput and has the potential to uncover novel cellular roles for genes. Our DNA microarray analysis demonstrated the up-regulation of C19orf2 and HSPA8 and the down-regulation of LGALS1, POU4F3, PSAP, AKT1, MBC2, and CACNA2D3 in all five of the ara-C-resistant cell lines compared to their respective parental cells (Table II). C19orf2 is known to mediate the function of a protein called RPB5, which binds to and negatively regulates the gene encoding the RNA polymerase II subunit 5 and which functionally antagonizes transcriptional modulation (26). HSPA8 is responsible for the maintenance of proper protein structure during stress (27). LGALS1 is a galectin 1 that mediates the induction of apoptosis, particularly in leukocytes (28). POU4F3 is a putative transcription factor associated with the differentiation of neurons and perhaps with the initiation of glioma (29). PSAP is a protein called prosaposin that has been shown to inhibit the process of tumor metastasis (30).
AKT1 is an isoform of AKT, a serine-threonine kinase that is targeted by PDGF-activated PI 3-kinase, which is involved in the regulation of diverse cellular functions including cell proliferation and apoptosis (31). MBC2 has a region of moderate similarity to a region of phosphoinositide-3-kinase class $2 \beta$ and is associated with glucose transport (32). CACNA2D 3 is a calcium channel, voltage-dependent, $\alpha 2 / \delta 3$ subunit, which is thought to have a tumor-suppressive function (33). These selected genes might thus be related to the development of ara-C resistance; however, the exact mechanisms by which they might function in ara-C resistance were not determined in this study.

Several studies have applied DNA microarray analysis to leukemic cells for evaluation of genes that modulate their sensitivity to ara-C (39-41). Takagaki et al examined the changes in the gene expression of leukemic cells after the cells were treated with ara-C (39). They showed the downregulation of chaperone genes in CEM cells, and the up-regulation of hemoglobin genes and the down-regulation of the aspartate synthetase gene in K562 cells. The genes identified in their study differed from the genes identified in our study. This discrepancy might be attributable to the difference in the experimental design, since Takagaki et al analyzed changes in the cells after exposure to the drug. Abe et al demonstrated up-regulation of the insulin-like growth factor I in an ara-C-resistant K562 variant (40). They did not detect a change in the AKT gene, which is downstream of insulin-like growth factor I. In contrast, the present study identified down-regulation of AKT1 and no alteration of insulin-like growth factor I. Although we have not further investigated this discrepancy, it may be due to differences in the degree of ara-C resistance between the two studies. Song et al detected a severe defect in the expression of dCK in two ara-C-resistant leukemic cell lines (41), which is considered to be one of the most likely mechanisms of ara-C resistance, whereas no change in $\mathrm{dCK}$ gene expression was detected in our microarray analysis. Thus, the results shown here are not fully consistent with those of previous studies. Nevertheless, the reproducibility of our microarray data is convincing, as the genes we extracted were identified in all five of the different ara-C-resistant cell lines.

Proteomic analysis has been successfully used to identify potential therapeutic targets and biomarkers of various cancers, including leukemia (42-44). However, proteomic analysis has not yet contributed information that would be useful for the improvement of the therapeutic outcome of AML. Here, using proteome analysis, we identified one gene, stathmin1, that was down-regulated in the CEM/ara-C subclone compared to the parental cell line. Stathmin1 is a microtubule-regulating protein that has an important role in the assembly and disassembly of the mitotic spindle. It has been shown to be involved in carcinogenesis, invasion, and metastasis of various cancers, including prostate, hepatocellular, and breast cancers $(34,35,45,46)$. Since the cytotoxicity of ara-C is cell cyclespecific, stathmin1 could potentially affect the action of ara-C on the cell cycle.

In conclusion, using conventional techniques as well as DNA microarray and proteome analyses, we have confirmed the involvement of known ara-C resistance-related factors and have identified new candidate resistance-related factors in 
ara-C-resistant leukemic cell lines. Given the importance of ara- $\mathrm{C}$ in treating leukemia, the present findings may offer a new strategy to overcome drug resistance and improve therapeutic outcomes.

\section{Acknowledgements}

We are indebted to Assistant Professor Yukie Tanaka (Department of Molecular Biology and Chemistry, Faculty of Medical Sciences, University of Fukui) for proteome analysis. This study was supported in part by Grants-in-Aid for Scientific Research from the Ministry of Education, Culture, Sports, Science, and Technology of Japan (No19591102, 2007-2009), a Grant from the Japanese Society of Clinical Pharmacology and Therapeutics (2007), Grants from the Gout Research Foundation (2007-2009), and the KYO prize for Leukemia Research from the Japan Leukemia Research Fund (2005).

\section{References}

1. Cheson BD: New antimetabolites in the treatment of human malignancies. Semin Oncol 19: 695-706, 1992.

2. Garcia-Carbonero R, Ryan DP and Chabner BA: Cytidine analogs. In: Cancer Chemotherapy and Biotherapy. Chabner BA and Longo DL (eds). Lippincott-Raven Publishers, Philadelphia, pp265-294, 1996.

3. Blum W and Marcucci G: New approaches in acute myeloid leukemia. Best Pract Res Clin Haematol 21: 29-41, 2008.

4. Tallman MS, Gilliland DG and Rowe JM: Drug therapy for acute myeloid leukemia. Blood 106: 1154-1163, 2005.

5. Fernandez HF and Rowe JM: Induction therapy in acute myeloid leukemia: intensifying and targeting the approach. Curr Opin Hematol 17: 79-84, 2010.

6. Wiley JS, Jones SP, Sawyer WH and Paterson AR: Cytosine arabinoside influx and nucleoside transport sites in acute leukemia. J Clin Invest 69: 479-489, 1982.

7. Galmarini CM, Jordheim L and Dumontet C: Role of IMP-selective 5 -nucleotidase (cN-II) in hematological malignancies. Leuk Lymphoma 44: 1105-1111, 2003.

8. Kufe DW, Major PP, Egan EM and Beardsley GP: Correlation of cytotoxicity with incorporation of ara-C into DNA. J Biol Chem 255: 8997-9990, 1980.

9. Kufe D, Spriggs D, Egan EM and Munroe D: Relationships among Ara-CTP pools, formation of (Ara-C)DNA, and cytotoxicity of human leukemic cells. Blood 64: 54-58, 1984.

10. Huang $P$ and Plunkett W: Fludarabine- and gemcitabine-induced apoptosis: incorporation of analogs into DNA is a critical event. Cancer Chemother Pharmacol 36: 181-188, 1995.

11. Yamauchi T, Ueda $\mathrm{T}$ and Nakamura $\mathrm{T}$ : A new sensitive method for determination of intracellular 1- $\beta$-D-arabinofuranosylcytosine 5 -triphosphate content in human materials in vivo. Cancer Res 56: 1800-1804, 1996.

12. Yamauchi T, Kawai Y, Kishi S, et al: Monitoring of intracellular $1-\beta$-D-arabinofuranosylcytosine 5'-triphosphate in 1- $\beta$-D-arabinofuranosylcytosine therapy at low- and conventional-doses. Cancer Sci 92: 546-553, 2001.

13. Plunkett W, Iacoboni S, Estey E, Danhauser L, Liliemark JO and Keating MJ: Pharmacologically directed ara-C therapy for refractory leukemia. Semin Oncol 12 (Suppl. 3): S20-S30, 1985.

14. Plunkett W, Liliemark JO, Adams TM, et al: Saturation of $1-\beta$-D-arabinofuranosylcytosine 5'-triphosphate accumulation in leukemia cells during high-dose 1- $\beta$-D-arabinofuranosylcytosine therapy. Cancer Res 47: 3005-3011, 1987.

15. Dumontet C, Fabianowska-Majewska K, Mantincic D, et al: Common resistance mechanisms to deoxynucleoside analogues in variants of the human erythroleukaemic line $\mathrm{K} 562$. Br J Haematol 106: 78-85, 1999

16. Lotfi K, Månsson E, Chandra J, et al: Pharmacological basis for cladribine resistance in a human acute $\mathrm{T}$ lymphoblastic leukaemia cell line selected for resistance to etoposide. Br J Haematol 113: 339-346, 2001.
17. Gandhi V, Estey E, Keating MJ and Plunkett W: Fludarabine potentiates metabolism of cytarabine in patients with acute myelogenous leukemia during therapy. J Clin Oncol 11: 116-124, 1993.

18. Estey E, Thall P, Andreeff M, et al: Use of granulocyte colonystimulating factor before, during, and after fludarabine plus cytarabine induction therapy of newly diagnosed acute myelogenous leukemia or myelodysplastic syndromes: comparison with fludarabine plus cytarabine without granulocyte colonystimulating factor. J Clin Oncol 12: 671-678, 1994.

19. Yue L, Saikawa Y, Ota K, et al: A functional single-nucleotide polymorphism in the human cytidine deaminase gene contributing to ara-C sensitivity. Pharmacogenetics 13: 29-38, 2003.

20. Yamauchi T, Negoro E, Kishi S, et al: Intracellular cytarabine triphosphate production correlates to deoxycytidine kinase/ cytosolic 5'-nucleotidase II expression ratio in primary acute myeloid leukemia cells. Biochem Pharmacol 77: 1780-1786, 2009.

21. Yong AS and Melo JV: The impact of gene profiling in chronic myeloid leukaemia. Best Pract Res Clin Haematol 22: 181-190, 2009.

22. Kaneta Y, Kagami Y, Katagiri T, et al: Prediction of sensitivity to STI571 among chronic myeloid leukemia patients by genomewide cDNA microarray analysis. Cancer Sci 93: 849-856, 2002.

23. Becker H, Marcucci G, Maharry K, et al: Favorable prognostic impact of NPM1 mutations in older patients with cytogenetically normal de novo acute myeloid leukemia and associated geneand microRNA-expression signatures: a Cancer and Leukemia Group B study. J Clin Oncol 28: 596-604, 2010.

24. Yamamoto S, Yamauchi T, Kawai Y, et al: Fludarabine-mediated circumvention of cytarabine resistance is associated with fludarabine triphosphate accumulation in cytarabine-resistant leukemic cells. Int J Hematol 85: 108-115, 2007.

25. Yoshio N, Kawai Y, Hori H and Ueda T: Resistance to 9-beta-Darabinofuranosyl-2-fluoroadenine due to reduced incorporation into DNA from competition by excess deoxyadenosine triphosphate: implications for different sensitivities to nucleoside analogues. Int J Hematol 81: 405-412, 2005.

26. Wei W, Dorjsuren D, Lin Y, et al: Direct interaction between the subunit RAP30 of transcription factor IIF (TFIIF) and RNA polymerase subunit 5 , which contributes to the association between TFIIF and RNA polymerase II. J Biol Chem 276: 12266-12273, 2001.

27. Craig EA, Weissman JS and Horwich AL: Heat shock proteins and molecular chaperones: mediators of protein conformation and turnover in the cell. Cell 78: 365-372, 1994.

28. Dhirapong A, Lleo A, Leung P, Gershwin ME and Liu FT: The immunological potential of galectin-1 and -3. Autoimmun Rev 8: 360-363, 2009.

29. Wu X, Rauch TA, Zhong X, Bennett WP, Latif F, Krex D and Pfeifer GP: $\mathrm{CpG}$ island hypermethylation in human astrocytomas. Cancer Res 70: 2718-2727, 2010.

30. Kang SY, Halvorsen OJ, Gravdal K, et al: Prosaposin inhibits tumor metastasis via paracrine and endocrine stimulation of stromal p53 and Tsp-1. Proc Natl Acad Sci USA 106: 12115-12120, 2009.

31. Santi SA and Lee H: The Akt isoforms are present at distinct subcellular locations. Am J Physiol Cell Physiol 298: C580-C591, 2010.

32. Lalioti V, Muruais G, Dinarina A, van Damme J, Vandekerckhove J and Sandoval IV: The atypical kinase Cdk5 is activated by insulin, regulates the association between GLUT4 and E-Syt1, and modulates glucose transport in 3T3-L1 adipocytes. Proc Natl Acad Sci USA 106: 4249-4253, 2009.

33. Wanajo A, Sasaki A, Nagasaki H, et al: Methylation of the calcium channel-related gene, CACNA2D3, is frequent and a poor prognostic factor in gastric cancer. Gastroenterology 135: 580-590, 2008.

34. Jeon TY, Han ME, Lee YW, et al: Overexpression of stathmin1 in the diffuse type of gastric cancer and its roles in proliferation and migration of gastric cancer cells. Br J Cancer 102: 710-718, 2010.

35. Hsieh SY, Huang SF, Yu MC, et al: Stathmin1 overexpression associated with polyploidy, tumor-cell invasion, early recurrence, and poor prognosis in human hepatoma. Mol Carcinog 49: 476-487, 2010.

36. Takemura H, Urasaki Y, Yoshida A, Fukushima T and Ueda T: Multaneous treatment with 1-beta-D-arabinofuranosylcytosine and daunorubicin induces cross-resistance to both drugs due to a combination-specific mechanism in HL60 cells. Cancer Res 61: 172-177, 2001. 
37. Lossos IS, Czerwinski DK, Alizadeh AA, et al: Prediction of survival in diffuse large-B-cell lymphoma based on the expression of six genes. N Engl J Med 350: 1828-1837, 2004.

38. Yeoh EJ, Ross ME, Shurtleff SA, et al: Classification, subtype discovery, and prediction of outcome in pediatric acute lymphoblastic leukemia by gene expression profiling. Cancer Cell 1: 133-143, 2002

39. Takagaki K, Katsuma S, Horio $\mathrm{T}$, et al: cDNA microarray analysis of altered gene expression in ara-C-treated leukemic cells. Biochem Biophys Res Commun 309: 351-358, 2003.

40. Abe S, Funato T, Takahashi S, et al: Increased expression of insulin-like growth factor $\mathrm{i}$ is associated with Ara-C resistance in leukemia. Tohoku J Exp Med 209: 217-228, 2006.

41. Song JH, Kim SH, Kweon SH, et al: Defective expression of deoxycytidine kinase in cytarabine-resistant acute myeloid leukemia cells. Int J Oncol 34: 1165-1171, 2009.

42. Lee IN, Chen $\mathrm{CH}$, Sheu JC, et al: Identification of human hepatocellular carcinoma-related biomarkers by two-dimensional difference gel electrophoresis and mass spectrometry. Proteome Res 4: 2062-2069, 2005.
43. Bhojwani D, Moskowitz N, Raetz EA and Carroll WL: Potential of gene expression profiling in the management of childhood acute lymphoblastic leukemia. Paediatr Drugs 9: 149-156, 2007.

44. Czibere A, Grall F and Aivado M: Perspectives of proteomics in acute myeloid leukemia. Expert Rev Anticancer Ther 6: 1663-1675, 2006.

45. Oishi Y, Nagasaki K, Miyata S, et al: Functional pathway characterized by gene expression analysis of supraclavicular lymph node metastasis-positive breast cancer. J Hum Genet 52: 271-279, 2007.

46. Chung MK, Kim HJ, Lee YS, et al: Hedgehog signaling regulates proliferation of prostate cancer cells via stathmin1. Clin Exp Med 10: 51-57, 2010 